\title{
Correction to: Comparison of Linear Versus Circular-Stapled Gastroenterostomy in Roux-en-Y Gastric Bypass: a Nationwide Population-Based Cohort Study
}

Marleen M. Romeijn ${ }^{1,2} \cdot$ Stijn van Hoef $^{1} \cdot$ Loes Janssen $^{1} \cdot$ Kelly G. H. van de Pas ${ }^{1,2} \cdot$ François M. H. van Dielen ${ }^{1}$ • Arijan A. P. M. Luijten ${ }^{1} \cdot K_{\text {Kevin W. A. Göttgens }}{ }^{1} \cdot$ Jan Willem M. Greve $^{2,3} \cdot$ Wouter K. G. Leclercq $^{1}$

(C) The Author(s) 2021

\section{Correction to: Obesity Surgery.}

https://doi.org/10.1007/s11695-021-05436-4

In Table 1, the headings in the top row are missing. The names in this heading represent the two groups of the study: CSA (left column) and LSA (right column).

The corrected table is shown below.

The online version of the original article can be found at https://doi.org/ $10.1007 /$ s $11695-021-05436-4$

Marleen M. Romeijn

m.romeijn@maastrichtuniversity.nl; Bariatric.resurge@mmc.nl

Stijn van Hoef

stijnvanhoef@gmail.com

Loes Janssen

Loes.Janssen@mmc.nl

Kelly G. H. van de Pas

kelly.van.de.pas@mmc.nl

François M. H. van Dielen

f.vandielen@mmc.nl

Arijan A. P. M. Luijten

A.Luijten@mmc.nl
Kevin W. A. Göttgens

Kevin.Goettgens@mmc.nl

Jan Willem M. Greve

j.greve@ zuyderland.nl

Wouter K. G. Leclercq

w.leclercq@mmc.nl

Department of Surgery, Máxima Medical Center, Veldhoven, The Netherlands

2 Research School NUTRIM, Department of Surgery, Maastricht University Medical Center, Maastricht, the Netherlands

3 Department of Surgery, Zuyderland Medical Center, Heerlen, the Netherlands 
Table 1 Baseline characteristics of the study population

\begin{tabular}{|c|c|c|c|}
\hline & $\mathrm{CSA}, n=881$ & LSA, $n=11,587$ & $p$ value \\
\hline \multicolumn{4}{|l|}{ Gender, no. (\%) } \\
\hline Female & $713(80.9)$ & $9541(82.3)$ & .291 \\
\hline Age (years) & $45.2 \pm 10.5$ & $45.1 \pm 10.6$ & .885 \\
\hline \multicolumn{4}{|l|}{ Preoperative comorbidities, no. (\%) } \\
\hline Hypertension & $333(37.8)$ & $4269(36.8)$ & .571 \\
\hline Type II diabetes mellitus & $143(16.2)$ & $2595(22.4)$ & $<.001 *$ \\
\hline Hyperlipidaemia & $169(19.2)$ & $2523(21.8)$ & .071 \\
\hline Gastroesophageal reflux disease & $89(10.1)$ & $1667(14.4)$ & $<.001 *$ \\
\hline OSAS & $265(30.1)$ & $2231(19.3)$ & $<.001 *$ \\
\hline Osteoarthritis & $344(39.0)$ & $5699(49.2)$ & $<.001 *$ \\
\hline Preoperative weight $(\mathrm{kg}, \pm \mathrm{SD})$ & $122.7 \pm 18.6$ & $122.7 \pm 17.9$ & .994 \\
\hline Preoperative BMI $\left(\mathrm{kg} / \mathrm{m}^{2} \pm \mathrm{SD}\right)$ & $42.7 \pm 4.9$ & $42.8 \pm 4.7$ & .265 \\
\hline Laparoscopic, no. (\%) & $879(99.8)$ & $11,573(99.9)$ & .396 \\
\hline Length of biliopancreatic $\operatorname{limb}(\mathrm{cm} \pm \mathrm{SD})$ & $72.9 \pm 15.3$ & $90.5 \pm 38.6$ & $<.001 *$ \\
\hline Length of alimentary limb $(\mathrm{cm} \pm \mathrm{SD})$ & $145.8 \pm 9.7$ & $133.6 \pm 33.7$ & $<.001 *$ \\
\hline Length of hospital stay (days \pm SD) & $2.3 \pm 1.7$ & $1.5 \pm 2.7$ & $<.001 *$ \\
\hline Number of readmission (<30 days), no. (\%) & $25(2.8)$ & $283(2.4)$ & .466 \\
\hline \multicolumn{4}{|l|}{ Postoperative complication <30 days, no. (\%) } \\
\hline CD grade I & $19(2.2)$ & $89(.8)$ & $<.001 *$ \\
\hline $\mathrm{CD}$ grade II & $25(2.8)$ & $99(.9)$ & $<.001 *$ \\
\hline CD grade IIIa & $3(.3)$ & $29(.3)$ & .610 \\
\hline $\mathrm{CD}$ grade IIIb & $41(4.7)$ & $382(3.3)$ & $.032 *$ \\
\hline $\mathrm{CD}$ grade IVa & $4(.5)$ & $15(.1)$ & $.017 *$ \\
\hline $\mathrm{CD}$ grade $\mathrm{IVb}$ & - & $9(.1)$ & .408 \\
\hline $\mathrm{CD}$ grade $\mathrm{V}$ & - & $1(.0)$ & .783 \\
\hline \multicolumn{4}{|l|}{ Type of complication, no. (\%) } \\
\hline Major bleeding & $21(2.4)$ & $136(1.2)$ & $.002 *$ \\
\hline Anastomotic leakage & $1(.1)$ & $43(.4)$ & .214 \\
\hline Intra-abdominal abscess & $2(.2)$ & $13(.1)$ & .343 \\
\hline Wound infection & $1(.1)$ & $14(.1)$ & .952 \\
\hline Intestinal obstruction & $1(.1)$ & $28(.2)$ & .447 \\
\hline Anastomotic stricture & $0(.0)$ & $1(.0)$ & .783 \\
\hline
\end{tabular}

Open Access This article is licensed under a Creative Commons Attribution 4.0 International License, which permits use, sharing, adaptation, distribution and reproduction in any medium or format, as long as you give appropriate credit to the original author(s) and the source, provide a link to the Creative Commons licence, and indicate if changes were made. The images or other third party material in this article are included in the article's Creative Commons licence, unless indicated otherwise in a credit line to the material. If material is not included in the article's Creative Commons licence and your intended use is not permitted by statutory regulation or exceeds the permitted use, you will need to obtain permission directly from the copyright holder. To view a copy of this licence, visit http://creativecommons.org/licenses/by/4.0/.

Publisher's Note Springer Nature remains neutral with regard to jurisdictional claims in published maps and institutional affiliations. 